\title{
Void-by-void versus multiple void interaction under mode I-mode II or mode I-mode III
} loading conditions

\author{
Andersen, R. G.; Nielsen, K. L.; Legarth, B. N.
}

Published in:

Engineering Fracture Mechanics

Link to article, DOI:

10.1016/j.engfracmech.2019.02.031

Publication date:

2019

Document Version

Peer reviewed version

Link back to DTU Orbit

Citation (APA):

Andersen, R. G., Nielsen, K. L., \& Legarth, B. N. (2019). Void-by-void versus multiple void interaction under mode I-mode II or mode I-mode III loading conditions. Engineering Fracture Mechanics, 214, 248-259. https://doi.org/10.1016/j.engfracmech.2019.02.031

\section{General rights}

Copyright and moral rights for the publications made accessible in the public portal are retained by the authors and/or other copyright owners and it is a condition of accessing publications that users recognise and abide by the legal requirements associated with these rights.

- Users may download and print one copy of any publication from the public portal for the purpose of private study or research.

- You may not further distribute the material or use it for any profit-making activity or commercial gain

- You may freely distribute the URL identifying the publication in the public portal 


\title{
Void-by-void versus multiple void interaction under mode I-mode II or mode I-mode III loading conditions
}

\author{
R.G. Andersen ${ }^{\mathrm{a}}$, K.L. Nielsen ${ }^{\mathrm{a}}$, B.N. Legarth ${ }^{\mathrm{a}}$ \\ ${ }^{a}$ Department of Mechanical Engineering, Solid Mechanics, Technical University of Denmark, \\ DK-2800 Kgs. Lyngby, Denmark
}

\begin{abstract}
Ductile crack initiation, governed by void growth to coalescence, is controlled by two distinct mechanisms, namely: (i) boid-by-void growth, where substantial growth of the void closest to the crack tip precedes growth of other voids, and (ii) multiple void interaction, where the voids grow simultaneously and interact in the vicinity of the crack tip prior to crack advance. Tvergaard and Hutchinson (2002) [Int. J. Solids and Struct, 39: 3581-3597] studied the two mechanisms in detail under far-field mode I loading conditions and demonstrated their interference by changing the size and spacing of discretely modeled voids. The present numerical work focuses on mixed mode loading conditions rather than limiting the study to mode I and takes up the question; how will a change in the far-field loading conditions affect the shift between the two mechanisms? A pre-existing straight crack with nearby discretely modeled voids in an elastic-plastic material is considered by a specialized 2D plane strain setting capable of out-of-plane displacements to allow for combinations of mode I, mode II, and mode III. Details of the void-by-void growth versus the multiple void interaction mechanisms are laid out for a range of load cases and geometrical void configurations, and increasing the mode mixity is found to favor void-by-void growth accompanied by a higher load intensity required on the far-field boundary to initiate the crack. The change between the two mechanisms is tied to a change in the deformation field that surrounds the voids, and the study reveals significant deformation that stretches above (and below) the row of discrete voids for increasing shear mode contributions yielding an increase in the void rotation.
\end{abstract}

Keywords: Ductile fracture, Fracture toughness, Void interaction, Mode mixity

URL: rgra@mek.dtu.dk (R.G. Andersen) 


\section{Nomenclature}

\begin{tabular}{|c|c|}
\hline$E$ & Young's modulus \\
\hline$f_{0}$ & Initial void volume fraction \\
\hline$g$ & Determinant of the metric tensor in the reference configuration \\
\hline$G$ & Determinant of the metric tensor in the current configuration \\
\hline$G_{s}$ & Elastic shear modulus \\
\hline$J$ & Far-field load intensity \\
\hline$K_{I}, K_{I I}, K_{I I I}$ & Stress intensity factors for mode I, mode II, and mode III, respectively \\
\hline$L^{i j k l}$ & Instantaneous moduli \\
\hline$N$ & Hardening exponent \\
\hline$r_{0}$ & Initial crack tip radius \\
\hline$R_{0}$ & Initial void radius \\
\hline$R_{f f}$ & Far-field radius \\
\hline$s_{i j}, s^{i j}$ & Covariant and contravariant components, respectively, of the Cauchy stress deviator \\
\hline$T^{i}$ & Surface tractions \\
\hline$u_{i}$ & Displacement in the $i$ th direction \\
\hline$x_{i}$ & Cartesian coordinates \\
\hline$X_{0}$ & Spacing between voids \\
\hline$\eta_{i j}$ & Lagrangian strain tensor \\
\hline$\theta_{\mathrm{II} / \mathrm{I}}, \theta_{\mathrm{III} / \mathrm{I}}$ & Mode mixity ratio for mode I-mode II and mode I-mode III, respectively \\
\hline$\nu$ & Poisson's ratio \\
\hline$\sigma$ & von Mises stress \\
\hline$\sigma_{i j}$ & Cauchy strain tensor \\
\hline$\sigma_{y}$ & Initial yield stress \\
\hline$\tau_{i j}$ & Kirchhoff stress tensor \\
\hline$\phi$ & Void rotation angle \\
\hline$\chi$ & Deformed ligament width \\
\hline$\chi_{0}$ & Initial ligament width \\
\hline$\psi$ & Angle to a material point on the far-field boundary \\
\hline
\end{tabular}




\section{Introduction}

The mechanism of ductile failure plays an important role in the assessment of structural integrity and several mechanisms, spanning multiple scales, are known to interact through a long sequence of events that eventually leads to material separation (Pineau et al., 2016). At severe plastic deformation, voids typically emerge from inclusions in the material either by debonding or particle cracking (see for instance Legarth, 2005; Benzerga et al., 2016). Upon further loading, the voids grow and coalesce to lead a propagating tearing crack. That is, the growth mechanism of the individual voids and their interaction are crucial to the ductile tearing process and of key importance to understand and enhance plate tearing toughness. Aravas and McMeeking (1985); Hom and McMeeking (1989) studied a blunt crack tip with a single void located immediately in front of the tip and exposed the setup to mode I loading under the assumption of small-scale yielding. Their results show the void growing primarily towards the crack tip and perpendicular to the applied mode I loading direction, which later has been confirmed in similar studies where a row of several voids was embedded in front of the crack tip. Gu (2000); Tvergaard and Hutchinson (2002); Kim et al. (2003) included six discretely modeled voids in their studies and revealed increasing crack resistance for decreasing initial void volume fraction. The mechanisms behind the change in crack resistance are that the voids grow individually with little influence from the neighboring voids (the so-called void-by-void mechanism) for a small initial void volume fraction in the homogenized material, whereas the voids grow simultaneously and neighboring voids interact (the so-called multiple void mechanism) for a large initial void volume fraction. This conclusion was reached for both circular cylindrical voids modeled in 2D plane strain (Tvergaard and Hutchinson, 2002) and for spherical voids modeled in 3D (Kim et al., 2003). Furthermore, Kim et al. (2003) concluded that model predictions based on the circular cylindrical $2 \mathrm{D}$ voids in plane strain underestimate the crack resistance compared to the 3D spherical voids - in particular for low values of the initial void volume fraction.

Several investigations have been dedicated to studying the effect of introducing additional rows of voids above and below the primary row immediately in front of the crack tip, changing the void shape of the discretely modeled voids, introducing second phase particles, and shifting the relative location of voided rows (Gao et al., 2005; 
Hütter et al., 2012, 2013). It has been demonstrated that by adding additional rows of voids above and below the primary row shield the voids in the vicinity of the tip (serving as the subsequent crack path) which results in stagnant crack growth and, hence, higher crack resistance. Srivastava et al. (2017) discovered several advantages of designing such microstructure and put forward a numerical demonstration of their findings by introducing a systematic wave formed pattern of voids that forces the crack to grow in a certain direction and thereby increasing the overall tearing toughness.

The main focus in the literature has been on mode I loading in an effort to bring out a clear picture of the mechanisms involved in ductile crack initiation and growth. Real life loading scenarios, however, comprise of mixed mode conditions and knowledge on the interaction from such conditions on a pre-existing crack path is limited. Roy and Narasimhan (1999) investigated the evolution of a single void embedded in front of a blunt crack tip under mixed mode I-mode II loading conditions and demonstrated significant void growth for pure mode I (which has been documented earlier) whereas the void growth eventually saturated for the highest mode mixity ratio considered. The present study takes the study of Roy and Narasimhan (1999), and the study of Tvergaard and Hutchinson (2002), one step further and focuses attention on a single row of several voids subjected to mixed mode loading conditions. The present work addresses; (i) the void growth and the related reduction in the ligaments between voids, and (ii) evaluate changes to the fracture toughness, accordingly, for mixed mode I-mode II and mixed mode I-mode III.

The paper is structured as follows. The problem at hand is outlined in Section 2, after which the material model is presented in Section 3 together with the numerical model. The obtained results are discussed in Section 4 and concluding remarks are given in Section 5.

\section{Problem formulation}

This study considers the problem illustrated in Fig. 1, modeled in the $x_{1} x_{2}$-plane under plane strain condition. The setup mimics the original problem considered in Tvergaard and Hutchinson (2002) but loads the blunt crack tip by mixed mode far-field loading conditions. Although the mixed mode loading conditions can be considered using antisymmetric boundary conditions, the entire domain is modeled here. The initial blunt 
crack tip has radius $r_{0}$, which is discretely modeled together with six circular cylindrical voids as depicted in Fig. 2. The radius of the individual voids is denoted $R_{0}$ and all voids are located along the $x_{1}$-axis at $x_{2}=0$. The relative spacing between two neighboring voids is $X_{0}$ (measured from void center to void center). For ligament $1, X_{0}$ is measured from the crack tip center to the center of void (1) (see Fig. 2). The domain boundary is modeled as being far from the crack tip by imposing $R_{f f}=10000 X_{0}$, where $R_{f f}$ is the outer radius. Thus, an assumption of small-scale yielding is valid.

The initial void volume fraction, $f_{0}$, of the homogenized material covering the vicinity of the individual voids is controlled by the void radius and void spacing as follows; $f_{0}=\pi\left(R_{0} / X_{0}\right)^{2}$ (defined in Tvergaard and Hutchinson, 2002) on which the initial crack tip radius, $r_{0}$, has no influence.

The present study will investigate pure mode I loading as well as mixed mode loading conditions comprising of mode I-mode II loading and mode I-mode III loading, respectively. The mixed mode loading conditions will be applied as linear combinations of the following far-field displacement components, $u_{i}$,

Pure mode I:

$$
\begin{aligned}
& u_{1}=\frac{K_{I}}{2 G_{s}} \sqrt{\frac{R_{f f}}{2 \pi}} \cos \left(\frac{\psi}{2}\right)\left[\kappa-1+2 \sin ^{2}\left(\frac{\psi}{2}\right)\right] \\
& u_{2}=\frac{K_{I}}{2 G_{s}} \sqrt{\frac{R_{f f}}{2 \pi}} \sin \left(\frac{\psi}{2}\right)\left[\kappa+1-2 \cos ^{2}\left(\frac{\psi}{2}\right)\right] \\
& u_{3}=0
\end{aligned}
$$

Pure mode II:

$$
\begin{aligned}
& u_{1}=\frac{K_{I I}}{2 G_{s}} \sqrt{\frac{R_{f f}}{2 \pi}} \sin \left(\frac{\psi}{2}\right)\left[\kappa+1+2 \cos ^{2}\left(\frac{\psi}{2}\right)\right] \\
& u_{2}=\frac{K_{I I}}{2 G_{s}} \sqrt{\frac{R_{f f}}{2 \pi}} \cos \left(\frac{\psi}{2}\right)\left[\kappa-1-2 \sin ^{2}\left(\frac{\psi}{2}\right)\right] \\
& u_{3}=0
\end{aligned}
$$

Pure mode III:

$$
\begin{aligned}
& u_{1}=u_{2}=0 \\
& u_{3}=\frac{2 K_{I I I}}{G_{s}} \sqrt{\frac{R_{f f}}{2 \pi}} \sin \left(\frac{\psi}{2}\right)
\end{aligned}
$$

where $K_{I}, K_{I I}$, and $K_{I I I}$ are the stress intensity factors for mode I, mode II, and mode III, respectively, $\kappa=3-4 \nu$ in plane strain, $G_{s}=E /(2(1+\nu))$ is the shear modulus, $E$ 
is Young's modulus, $\nu$ is Poisson's ratio, and $\psi \in[-\pi ; \pi]$ is the angle to the material point on the far-field boundary ( $\psi=0$ coincides with the $x_{1}$-axis and positive counterclockwise, see Fig. 1). The total far-field load intensity will be quantified through the stress intensity factors for the three individual fracture modes in the following manner:

$$
J=K_{I}^{2}\left(\frac{1-\nu^{2}}{E}\right)+K_{I I}^{2}\left(\frac{1-\nu^{2}}{E}\right)+K_{I I I}^{2}\left(\frac{1+\nu}{E}\right) .
$$

In the presentation of results, this far-field quantity is normalized by the initial yield stress, $\sigma_{y}$, and the initial void spacing, $X_{0}$. Throughout, for the results on mode I-mode II loading $K_{I I I}=0$, while for mode I-mode III loading $K_{I I}=0$.

The loading is applied by increasing the far-field stress intensity factors monotonically from zero. During loading, the void related deformation simultaneously yields a deformation of the ligaments between the voids which is largely dependent on the farfield mode mixity. The focus is on this deformation and the crack is assumed to initiate growth when the ligament between the initial blunt crack tip and void (1) (ligament 1 ) has reduced to a critical width of $\chi / \chi_{0}=0.5$ (as in Tvergaard and Hutchinson, 2002). A generic representation is presented in Fig. 3, where $\chi_{0}$ defines the undeformed ligament width and $\chi$ defines the width of the same ligament but in its deformed configuration. Both $\chi_{0}$ and $\chi$ are determined as the projected distance parallel to the $x_{1}$-axis between the two highlighted nodes in Fig. 3. These two nodes are chosen such that, in the undeformed configuration, the distance between them is the smallest possible. Displacements along the $x_{2}$-direction are not taken into account when calculating $\chi_{0}$ and $\chi$. It should be mentioned that the approach taken to calculate the ligament reduction is only suitable for the low contributions of the shear modes considered. A relative increase in the shear contribution may deform the ligaments such that the shortest distance can no longer be approximated by the horizontal distance.

In addition to the void growth, the void rotation denoted $\phi$ is considered as an indication of the level of deformation going into the material surrounding the voids (see Fig. 4). The angle is defined solely in the $x_{1} x_{2}$-plane for the mode I-mode II load case by the rotation of the dashed line connecting the node at the top and bottom of the void in the undeformed state (see Fig. 4a). The mode I-mode III load case is treated in a similar manner, but solely in the $x_{2} x_{3}$-plane (see Fig. 4b). The embedded single row of voids is located in the desired path for a pure mode I crack. However, this is not necessarily the desired path for a mixed mode crack as it might deviate from 
a straight crack path in an isotropic solid. The shear mode contribution considered in this study is, however, small and hence it is reasonable to approximate the crack path as straight. Furthermore, the choice of only including one single row of voids is to investigate the fundamentals of mode mixity on crack tip void interaction.

\section{Model: constitutive relations and finite element formulation}

\section{1. $J_{2}$-flow theory}

An isotropic elastic-plastic material following $J_{2}$-flow theory is employed in the computations. Finite deformations are accounted for and the uni-axial true stress-logarithmic strain relation takes the form

$$
\varepsilon= \begin{cases}\frac{\sigma}{E} & \text { for } \sigma<\sigma_{y} \\ \frac{\sigma_{y}}{E}\left(\frac{\sigma}{\sigma_{y}}\right)^{1 / N} & \text { for } \sigma \geq \sigma_{y}\end{cases}
$$

where $E$ is Young's modulus, $\sigma_{y}$ is the initial yield stress, $N$ is the strain hardening exponent, and $\sigma=\sqrt{3 / 2 s^{i j} s_{i j}}$ is the von Mises effective stress, where $s_{i j}$ is the Cauchy stress deviator. From Eq. (2), the tangent modulus is defined by $E_{t}=\mathrm{d} \sigma / \mathrm{d} \varepsilon$. In this work, a total Lagrangian formulation is used within a hypo-elastic framework, assuming small elastic strains but large plastic strains. In this formulation, $\eta_{i j}$ is the Lagrangian strain tensor given as $\eta_{i j}=\frac{1}{2}\left(u_{i, j}+u_{j, i}+u_{, i}^{k} u_{k, j}\right)$. The incremental relationship between Kirchhoff stresses, $\tau_{i j}$ and Lagrangian strains is

$$
\dot{\tau}^{i j}=L^{i j k l} \dot{\eta}_{k l}
$$

where $L^{i j k l}$ are the instantaneous moduli taken from Hutchinson (1973), and the total strain increment decomposes into an elastic part, $\dot{\eta}_{i j}^{e}$, and a plastic part, $\dot{\eta}_{i j}^{p}$, respectively. Here, with the plastic strain increment being calculated on basis of the Jaumann rate of the Cauchy stress tensor and the Cauchy stress deviator. The relation between the Kirchhoff and Cauchy stress tensor is $\sigma^{i j}=\sqrt{G / g} \tau^{i j}$, where $g$ and $G$ are the determinant of the metric tensor in the reference configuration and current deformed configuration, respectively. The principle of virtual work is on incremental form described as

$$
\int_{V}\left(\dot{\tau}^{i j} \delta \eta_{i j}+\tau^{i j} \dot{u}_{, i}^{k} \delta u_{k, j}\right) \mathrm{d} V=\int_{S} \dot{T}^{i} \delta u_{i} \mathrm{~d} S
$$


where $\tau^{i j}$ is the Kirchhoff stress tensor, $u_{i}$ is the deformation tensor and $T^{i}$ are the surface tractions. The left-hand side is integrated over the volume $V$ whereas the right-hand side is integrated over the surface $S$, both in the undeformed configuration. A comprehensive description of the governing equations can be found in Tvergaard (1990).

\subsection{Finite element formulation}

All analyses are carried out using a quasi-static, finite element framework built into an in-house Fortran code. The material domain, depicted in Fig. 1, is discretized using a special eight-node 2D plane strain element with an additional nodal degree of freedom to account for out-of-plane deformations. The purpose-built element is described in Andersen et al. (2018), sustaining the plane strain condition by enforcing the total out-of-plane strain component, $\eta_{33}$, to zero. Shortly described, the basis of the element is a 20-node 3D brick element, but here collapsed in the $x_{3}$-direction such that it has no physical extension in this direction and all coinciding nodes are merged. All dependencies of the displacement gradients in the $x_{3}$-direction are thereby written out of the element formulation. This gives the opportunity to investigate the desired mixed mode load cases without introducing computationally costly 3D elements with constraint equations.

Figure 5 displays the discretization in the vicinity of the voids, here for the model

parameter set of $r_{0} / X_{0}=1 / 10$ and $R_{0} / X_{0}=1 / 15$. For all models, a fine mesh area consisting of 7960 elements in the path of the crack is used to capture the void expansion, the thinning of the ligaments, and the resulting crack initiation. The mesh is coarsened in the radial direction towards the far-field boundary in order to reduce the total number of elements without compromising the accuracy of results. A total of 9526 elements with 88,035 degrees of freedom are used.

\section{Results}

The initial void spacing, $X_{0}$, is kept constant throughout, while both the initial crack tip radius, $r_{0}$, and the initial void radius, $R_{0}$, are subject to a parameter study in the 
range (here normalized with $X_{0}$ )

$$
\begin{gathered}
r_{0} / X_{0} \in[1 / 30 ; 1 / 10] \\
R_{0} / X_{0} \in[1 / 60 ; 1 / 15]
\end{gathered}
$$

where the latter yields an initial void volume fraction in the range: $f_{0} \in[0.000873 ; 0.014]$. The choice of parameter intervals is adopted from Tvergaard and Hutchinson (2002) to facilitate a direct comparison of results. Pure mode I loading is considered to form a basis for comparison, while the primary focus is on mixed mode loading conditions with a dominant mode I component. The ratio between the shearing mode (mode II or mode III) to the normal mode (mode I) is in the range of

$$
\begin{gathered}
\theta_{\mathrm{II} / \mathrm{I}} \in[0 ; 0.5] \\
\theta_{\mathrm{III} / \mathrm{I}} \in[0 ; 0.5]
\end{gathered}
$$

where $\theta_{\mathrm{II} / \mathrm{I}}=K_{I I} / K_{I}$ and $\theta_{\mathrm{III} / \mathrm{I}}=K_{I I I} / K_{I}$, respectively. The mode mixity ratios are, throughout, chosen sufficiently low such that voids remain open rather than collapsing, as would be expected for intense mode II or mode III. All material parameters used in the following are listed in Table 1 and resembles ductile medium-strength steel.

To demonstrate the deformation inflicted by the individual load cases, Fig. 6 presents the deformation contours for a setup with an initial crack tip radius of $r_{0} / X_{0}=1 / 30$ and an initial void volume fraction of $R_{0} / X_{0}=1 / 15\left(f_{0}=0.014\right)$. Each subfigure shows, respectively, the pure mode I load case, the mode I-mode II load case with a ratio of $\theta_{\mathrm{II} / \mathrm{I}}=0.2$, and the mode I-mode III load case with a ratio of $\theta_{\mathrm{III} / \mathrm{I}}=0.2$. The applied far-field load intensity is approximately $J /\left(\sigma_{y} X_{0}\right) \approx 0.5$ in all contour plots, with $J$ calculated from Eq. (1). The deformation is concentrated near the voids (disregarding deformation in the $u_{3}$-direction) for pure mode I loading, whereas it spreads largely above and below the voids for mode I-mode II and mode I-mode III, respectively. Since the far-field load intensity is equal in the three load cases, the mode I contribution will be different and the results clearly underline that mode I loading is responsible for void growth while the shearing modes are responsible for shear deformation in the material surrounding the voids. The out-of-plane deformation, $u_{3}$, (in the $x_{3}$-direction) is depicted in Fig. $6 \mathrm{c}$ and exists only in the mode I-mode III load case. 
The first investigation focuses on how the initial crack tip radius influences the farfield load intensity (quantified by the relation in Eq. (1)) required to deform ligament 1 to a level of $\chi / \chi_{0}=0.5$. Figure 7 depicts with asterisks the applied far-field load intensity in pure mode $\mathrm{I}\left(\theta_{\mathrm{II} / \mathrm{I}}=\theta_{\mathrm{III} / \mathrm{I}}=0\right)$ as a function of the initial void volume fraction, $f_{0}$, for two different initial crack tip radii, $r_{0} / X_{0}=1 / 30$ (Fig. 7a) and $r_{0} / X_{0}=1 / 10$ (Fig. $7 \mathrm{~b}$ ). Also depicted in both figures is a reference curve, shown as a dotted line, which has been extracted from the work of Tvergaard and Hutchinson (2002) for the case of $r_{0} / X_{0}=1 / 10$. A good agreement with Tvergaard and Hutchinson (2002) is obtained for the same parameter set (see Fig. 7b) and the exponential-like increase in far-field load intensity, and thereby also the increase in fracture toughness, is reproduced. Comparing Figs. 7a and 7b, a small increase in far-field load intensity is revealed in the former for the decrease in initial crack tip radius going from $r_{0} / X_{0}=$ $1 / 10$ to $r_{0} / X_{0}=1 / 30$.

The exponential-like increase in far-field load intensity for decreasing initial crack tip radius also exists when applying a shearing mode to the far-field boundary. Figure 7 presents additionally the far-field load intensity for a mode mixity ratio of $\theta_{\mathrm{II} / \mathrm{I}}=0.2$ (mode I-mode II) and $\theta_{\mathrm{III} / \mathrm{I}}=0.2$ (mode I-mode III) required to reach $\chi / \chi_{0}=0.5$. For each value of initial void volume fraction, an increase in fracture toughness is evident when applying a mixed mode loading condition, although the effect is dependent on both the mode mixity and initial void volume fraction. Both mode I-mode II and mode I-mode III load cases display nearly the same toughness for the largest initial void volume fraction $\left(f_{0}=0.014\right)$, whereas the mode I-mode III load case displays the largest fracture toughness at the lower initial void volume fraction $\left(f_{0}=0.000873\right)$. This difference in fracture toughness between loading conditions is more distinct for the smaller initial crack tip radius (compare Fig. $7 \mathrm{a}$ and $7 \mathrm{~b}$ ).

The far-field load intensity determined by Eq. (1), and employed in Fig. 7, takes into account the contributions from both mode I, mode II and mode III. However, disregarding mode II and mode III contributions, and thereby only considering the mode I contribution, for the mode mixity load cases will not lower the far-field load intensity to the level predicted for pure mode I (see Fig. 7). Thus, a shielding effect on the void growth originates from the far-field shear mode contributions yielding delayed crack initiation. 
The fracture toughness dependency on the loading conditions is directly linked to the void expansion presented in Fig. 8. Here, the relative volume expansions $V / V_{0}$ for void (1) and (2) are shown for an initial crack tip radius of $r_{0} / X_{0}=1 / 10$ and an initial void volume fraction of $R_{0} / X_{0}=1 / 60\left(f_{0}=0.000873\right)$. In the pure mode I load case, depicted with a thin solid line, void (1) grows rapidly. In contrast, the volume of the first void evolves slower when a shear mode is applied (with mode I-mode III being slowest), cementing that mode I drives void growth. It should, however, be noted that due to the plane strain assumption only the displacements in the $x_{1} x_{2}$-plane is taken into account when calculating the void volume, thus it yields a projected image of the void growth in the case of mode I-mode III loading (leaving out the $u_{3}$-displacements in the volume calculations). The slower evolution in the void volume for mixed mode loading conditions also manifests itself through a higher toughness (see Fig. 7). The void-by-void growth mechanism here reveals itself by void (2) showing insignificant growth in the range of the far-field load intensity considered. To compare with earlier studies, a reference curve from the work by Tvergaard and Hutchinson (2002) (thick solid lines) is included in Fig. 8.

To focus attention on the influence of mode mixity on the transition from voidby-void growth to multiple void interaction, the remaining study is limited to the smallest crack tip radius $\left(r_{o} / X_{0}=1 / 30\right)$. Throughout, a crack will be assumed to initiate whenever $\chi / \chi_{0}=0.5$ in ligament 1 (see e.g. Benzerga, 2002). At this level of deformation, the elements near the free surface of the voids, and near the initial blunt crack tip, still maintain a decent aspect ratio and no remeshing is employed.

\subsection{Deformation of ligament 1 and void (1)}

Figure 9 shows the relationship between the applied far-field load intensity, $J /\left(\sigma_{y} X_{0}\right)$, and the reduction of ligament 1 , $\chi / \chi_{0}$, for $r_{0} / X_{0}=1 / 30$ and $R_{0} / X_{0}=1 / 60$ in Fig. 9 a and $R_{0} / X_{0}=1 / 15$ in Fig. 9b, respectively. In line with Fig. 7a, an increased mode mixity ratio requires higher far-field load intensity to thin ligament 1 to $\chi / \chi_{0}=0.5$, which in turn signals higher toughness. That is, the far-field load intensity at which a crack will initiate is highly dependent on both the initial void volume fraction, the mode mixity ratio, and the type of mode mixity. It is evident that a large far-field load intensity is necessary to thin ligament 1 and thereby cause crack initiation $\left(\chi / \chi_{0}=\right.$ 0.5) for the low initial void volume fraction considered in Fig. 9a. This can be assigned 
to the void-by-void growth mechanism, where only one void grows at a time, essentially limiting the deformation to the first ligament. In contrast, crack initiation occurs at a lower far-field load intensity for the larger initial void volume fraction (see Fig. 9b), which results in a more compliant material response and a lower fracture toughness. The easier crack initiation is tied to the multiple void interaction mechanism which causes several ligaments to reduce simultaneously. Independent of the initial void volume fraction, however, the far-field load intensity at crack initiation is significantly increased by introducing mode mixity loading which again indicates that a major part of the energy from the shearing modes are going into the surrounding material rather than going into reducing ligament 1 . The diffuse dissipation of energy in the material above (and below) the row of voids is not observed to the same extent for the pure mode I loading.

The rotation of void (1) is considered in the following to investigate the influence of mode mixity as the material above and below the row of voids seems to deform additionally when increasing mode mixity. In the same manner as Fig. 9, the rotation angle, $\phi$, of void (1) (see also Fig. 4) is depicted in Fig. 10 as a function of the reduction of ligament $1, \chi / \chi_{0}$. For a low initial void volume fraction, Fig. 10a displays a rotation largely dependent on both the type of mode mixity and the mode mixity ratio. For example, the rotation of void (1) reaches $40^{\circ}$ (the most extreme case) for the largest mode I-mode II ratio considered, whereas the void rotation is only $33^{\circ}$ for the largest mode I-mode III ratio. It is also observed from Fig. 10 that the amount of void rotation is clearly dependent on the two types of mode mixity for the lowest initial void volume fraction, whereas the difference diminishes for larger initial void volume fractions (see Fig. 10b). It is also worth to note that void (1) obtains the largest rotation when subject to mode I-mode III loading at low mode mixity ratios when compared to mode I-mode II loading. However, this changes when the mode mixity ratio increases as the rotation under mode I-mode II loading exceeds that of mode I-mode III.

A relationship between the rotation, $\phi$, for void (1) and the mode mixity ratio, $\theta$, for both types of mode mixity and fixed energy dissipation is brought out in Fig. 11. Here, considering a relatively small far-field load intensity at $J /\left(\sigma_{y} X_{0}\right)=0.25$, so that crack initiation has not occurred and $\chi / \chi_{0}>0.5$ in all cases. It is deducted that lower contributions of the shear mode (either mode II or mode III) will reduce void rotation 
and concentrate the deformation in ligament 1 , whereas the void rotation increases for higher shear contributions and spread large parts of the energy dissipation to the material surrounding the row of voids. The relationship between the rotation and the mode mixity ratio is, however, not the same for the two types of mode mixity. A highly non-linear relation is predicted for mode I-mode II loading with the mode mixity ratio having almost no influence on the void rotation for low shear contributions, whereas the void rotation increases dramatically as the mode mixity ratio increases. On the other hand, a nearly linear relation between the mode mixity ratio and the void rotation is predicted for the mode I-mode III loading condition.

\subsection{Deformation of ligaments 1 through 6 and voids (1) through (6)}

Crack initiation has until this point been examined by investigating the reduction of ligament 1 through the criterion $\chi / \chi_{0}=0.5$. Next, the reductions of all ligaments (1 through 6 ) are subject of investigation and the interaction between voids is highlighted. Figure 12 presents all six ligament reductions at the far-field load intensity required for ligament 1 to reduce to $\chi / \chi_{0}=0.5$ (see Fig. 9 for the specific far-field load intensity for each mode mixity case). Here, the two extreme cases for the initial void volume fraction are considered. Figure 12a depicts clearly the void-by-void growth mechanism for low initial void volume fraction (see also in Fig. 8), where almost no deformation is present in the ligaments other than the first two. In fact, the reductions of the individual ligaments are nearly identical for all mode mixity ratios and type of mode mixity considered. Only the case of $\theta_{\mathrm{II} / \mathrm{I}}=0.5$ displays some deformation of all ligaments for low initial void volume fraction.

Figure $12 \mathrm{~b}$ displays severe deformation in all of the ligaments for all mode mixities. Here, considering the large initial void volume fraction. This is in line with the findings in Tvergaard and Hutchinson (2002), where interaction between multiple voids was found for larger initial void volume fractions. The multiple void interaction is particularly evident for load cases with small shear contributions. But, a tendency towards a shift in the mechanism from multiple void interaction to void-by-void growth is seen for higher ratios as the deformation intensifies in ligament 1, while diminishing in all others. This suggests that for even higher mode mixity ratios, the void-by-void growth mechanism could well be achieved. It is, however, important to emphasize that the far-field load intensity, $J /\left(\sigma_{y} X_{0}\right)$, is not the same across the mode mixity ratios and 
the type of mode mixity depicted in Fig. 12. In fact, a great difference is predicted in the required far-field load intensity to reach a deformation in ligament 1 of $\chi / \chi_{0}=0.5$ (also evident from Fig. 9b).

Figure 13 depicts the rotation of all six voids, at the fixed far-field load intensity $J /\left(\sigma_{y} X_{0}\right)=0.5$, for different mode mixity ratios. Figure 13a considers the smallest initial void volume fraction $\left(f_{0}=0.000873\right)$ and shows a significant variation of the rotation between the voids, where void (1) rotates much more than the remaining five voids. This is tied to the void-by-void growth mechanism. However, by comparing Fig. 12a to Fig. 13a it is seen that ligament 2 through 6 remain nearly undeformed, while the related voids undergo rotations. Thus, the increased deformation above and below the row of voids introduced by the shear modes primarily affects the void rotation.

Figure 13b considers a case of large initial void volume fraction and displays a significant change in the void rotation when compared to the corresponding case of lower initial void volume fraction (compare to Fig. 13a). The rotation is evident for both mode I-mode II and mode I-mode III loading conditions which, in turn, ties to the multiple void interaction mechanism being active. This is especially true for low mode mixity ratios and nearly equal rotation for all voids $\left(\phi=5^{\circ}\right)$ is predicted for the lowest mode mixity ratios $\left(\theta_{\mathrm{II} / \mathrm{I}}=\theta_{\mathrm{III} / \mathrm{I}}=0.1\right)$. However, the approximately equal rotation of all voids ceases as the mode mixity ratio increases and for $\theta_{\mathrm{II} / \mathrm{I}}=0.2$, and above, the mode I-mode II loading condition approaches the void-by-void growth mechanism. The same case is predicted for the mode I-mode III loading condition but only for $\theta_{\mathrm{III} / \mathrm{I}}=0.5$. The tendency of a decreasing void rotation for low mode mixity ratio, however, may be an artifact of only including six discrete voids in the model.

\section{Concluding remarks}

The void interaction that takes place in front of an initial blunt crack tip is investigated with focus on how mixed mode loading conditions affect the shift from void-by-void growth to multiple void interaction observed by Tvergaard and Hutchinson (2002) under mode I loading. A specialized 2D plane strain, finite strain, finite element model is employed with six discretely modeled voids embedded in front of the initial crack tip. Two types of mode mixity are considered, these being; mode I-mode II and mode 
I-mode III loading, respectively, with mode I as the dominant loading component. The key findings are;

- Mode I-mode II loading condition favors a shift towards the void-by-void growth mechanism for sufficiently large mode II contribution $\left(\theta_{\mathrm{II} / \mathrm{I}}>0.2\right)$, even for a large initial void volume fraction (see Fig. 12). This is tied to the competition between the mode II contribution, that attempts closing the voids, and the mode I contribution that opens the voids. Thus, an increased far-field load intensity is required compared to mode I loading to thin the first ligament sufficiently to achieve crack initiation. The increased load intensity contributes to a deformation spread out above and below the ligaments and as a result, severe in-plane void rotation is predicted for increased mode II contributions (see Figs. 10 and 13). Similarly, the void rotation seems to follow the void growth mechanism with a shift from void-by-void rotation to multiple void rotation as the initial void volume fraction increases. However, this is highly dependent on the mode mixity ratio with large ratios favoring multiple void rotation.

- Mode I-mode III loading condition has a similar influence on the overall response as predicted for the mode I-mode II loading condition. However, the mode III loading does not involve the same void closing effect and, thus, the competition between the opening mode and shear mode is essentially absent. This is also evident from Fig. 11, where a nearly linear relation is predicted between the outof-plane void rotation (in the $x_{2} x_{3}$-plane) and the prescribed mode mixity ratio. The void rotation, however, originates from a shear deformation of the material above (and below) the row of voids, and this leads to an increase in the far-field load intensity required to initiate the crack (see Fig. 9). The introduced mode III loading is found to favor a shift from multiple void interaction towards voidby-void growth for the largest initial void volume fraction as well as the highest mode mixity ratio considered (see Fig. 12). In the same fashion, a shift from multiple voids rotation towards void-by-void rotation is predicted (see Fig. 13).

The findings for mixed mode loading conditions are in line with the original study by Tvergaard and Hutchinson (2002), where the void-by-void growth mechanism exists for low initial void volume fractions, while multiple void interaction sets in for materials 
with a high initial void volume fraction. However, a shift from multiple void interaction to void-by-void growth is predicted for large initial void volume fractions when the shear contribution is increased.

\section{Acknowledgments}

The work is financially supported by the Department of Mechanical Engineering at the Technical University of Denmark in the project "Advancing Numerical Analysis of Large Scale Crack Propagation in Plate Structures".

\section{References}

Andersen, R. G., Woelke, P. B., Nielsen, K. L., 2018. Cohesive traction-separation relations for plate tearing under mixed mode loading. European Journal of Mechanics - A/Solids 71, 199-209.

Aravas, N., McMeeking, R. M., 1985. Finite-element analysis of void growth near a blunting crack tip. Journal of the Mechanics and Physics of Solids 33 (1), 25-49.

Benzerga, A. A., 2002. Micromechanics of coalescence in ductile fracture. Journal of the Mechanics and Physics of Solids 50 (6), 1331-1362.

Benzerga, A. A., Leblond, J. B., Needleman, A., Tvergaard, V., 2016. Ductile failure modeling. International Journal of Fracture 201 (1), 29-80.

Gao, X., Wang, T., Kim, J., 2005. On ductile fracture initiation toughness: Effects of void volume fraction, void shape and void distribution. International Journal of Solids and Structures 42 (18-19), 5097-5117.

Gu, I., 2000. Finite element analyses of deformation around holes near a crack tip and their implications to the j-resistance curve. Fatigue and Fracture of Engineering Materials and Structures 23 (11), 943-952.

Hom, C. L., McMeeking, R. M., 1989. Three-dimensional void growth before a blunting crack tip. Journal of the Mechanics and Physics of Solids 37 (3), 395-415.

Hutchinson, J. W., 1973. Finite Strain Analysis of Elastic-Plastic Solids and Structures. 
Hütter, G., Zybell, L., Mühlich, U., Kuna, M., 2012. Ductile crack propagation by plastic collapse of the intervoid ligaments. International Journal of Fracture 176 (1), $81-96$.

Hütter, G., Zybell, L., Mühlich, U., Kuna, M., 2013. Consistent simulation of ductile crack propagation with discrete $3 \mathrm{~d}$ voids. Computational Materials Science 80, 61-70.

Kim, J., Gao, X., Srivatsan, T., 2003. Modeling of crack growth in ductile solids: a three-dimensional analysis. International Journal of Solids and Structures 40 (26), $7357-7374$.

Legarth, B. N., 2005. Effects of geometrical anisotropy on failure in a plastically anisotropic metal. Engineering Fracture Mechanics 72 (18), 2792-2807.

Pineau, A., Benzerga, A. A., Pardoen, T., 2016. Failure of metals I: Brittle and ductile fracture. Acta Materialia 107, 424-483.

Roy, Y., Narasimhan, R., 1999. A finite element investigation of the effect of crack tip constraint on hole growth under mode i and mixed mode loading. International Journal of Solids and Structures 36 (10), 1427-1447.

Srivastava, A., Osovski, S., Needleman, A., 2017. Engineering the crack path by controlling the microstructure. Journal of the Mechanics and Physics of Solids 100 (December 2016), 1-20.

Tvergaard, V., 1990. Material failure by void growth to coalescence. Advances in applied mechanics 27, 83-151.

Tvergaard, V., Hutchinson, J. W., 2002. Two mechanisms of ductile fracture: Void by void growth versus multiple void interaction. International Journal of Solids and Structures 39 (13-14), 3581-3597.

\section{Tables}


Table 1: Material properties.

\begin{tabular}{lll}
\hline Parameters & Notation & Value \\
\hline Young's modulus & $E$ & $200 \mathrm{GPa}$ \\
Poisson's ratio & $\nu$ & 0.3 \\
Yield stress & $\sigma_{y}$ & $600 \mathrm{MPa}$ \\
Hardening exponent & $N$ & 0.1 \\
\hline
\end{tabular}




\section{Figures}

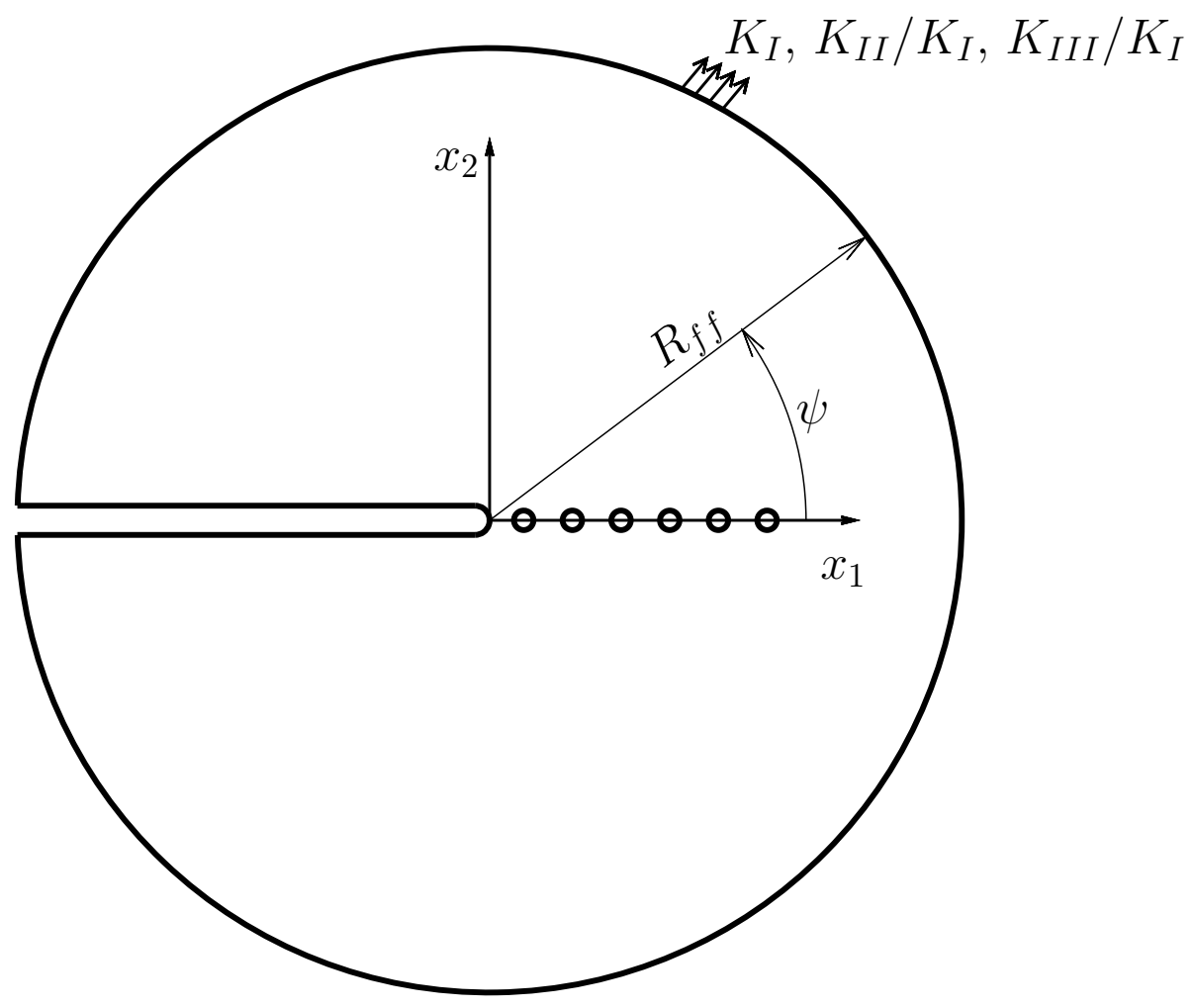

Figure 1: Schematic of the 2D plane strain, small-scale yielding model. Here, $R_{f f}$ is the far-field radius and the $x_{3}$-component is out-of-plane (not shown). The far-field boundary loading corresponds to either mode I loading, mixed mode I-mode II loading, or mixed mode I-mode III loading.



Figure 2: Schematic of the discretely modeled voids. Here, $X_{0}$ is the center-to-center void spacing, $r_{0}$ is the initial radius of the blunt crack tip, and $R_{0}$ is the initial radius of the voids. The circled numbers indicate void number and the framed numbers indicate ligament number. 


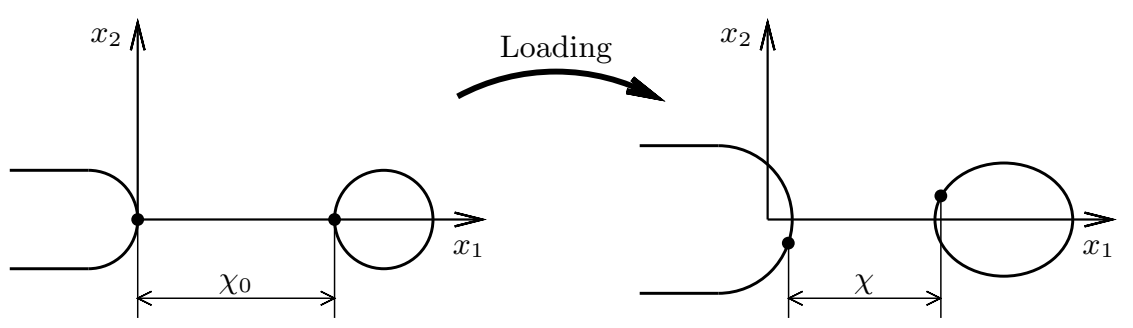

Figure 3: Generic representation of the ligament reduction, $\chi / \chi_{0}$.



(a)

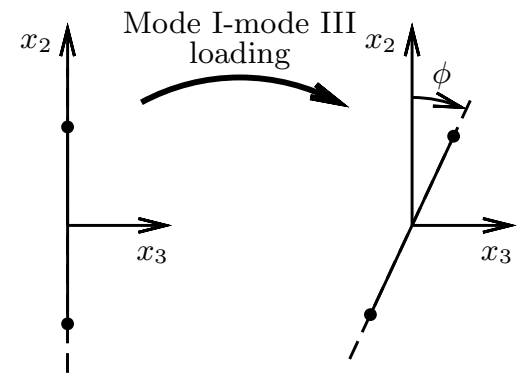

(b)

Figure 4: Generic representation of the void rotation, $\phi$, for (a) mode I-mode II loading and (b) mode I-mode III loading.

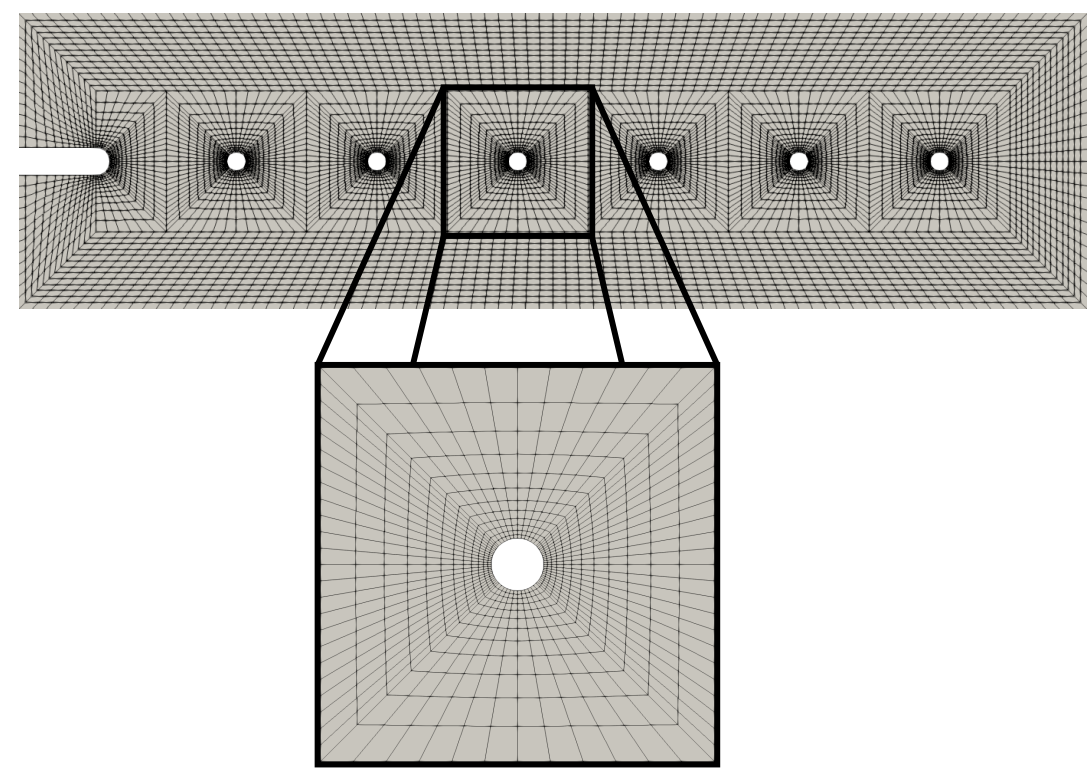

Figure 5: Mesh discretization method for the domain near the crack tip and voids. Here shown for the parameter set of $r_{0} / X_{0}=1 / 10$ and $R_{0} / X_{0}=1 / 15\left(f_{0}=0.014\right)$. 


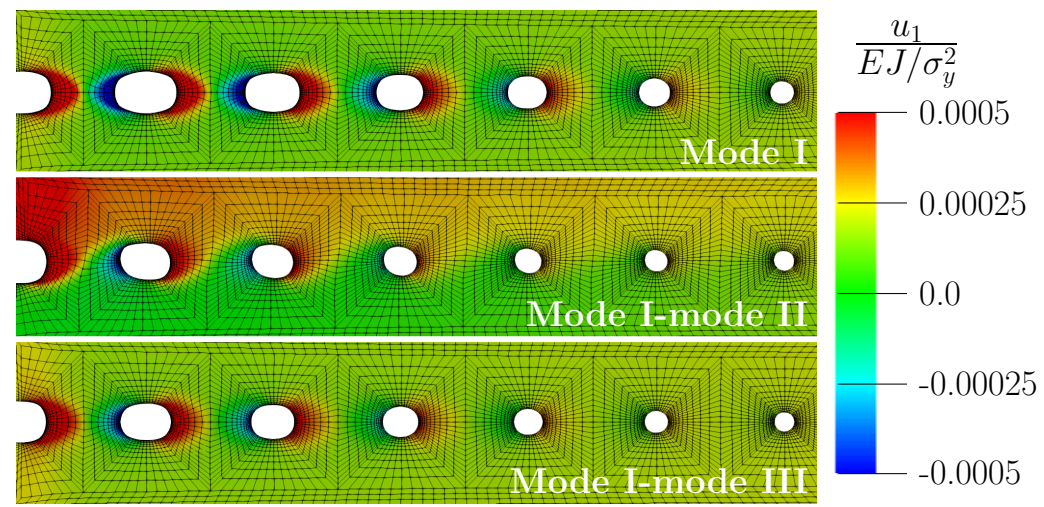

(a)

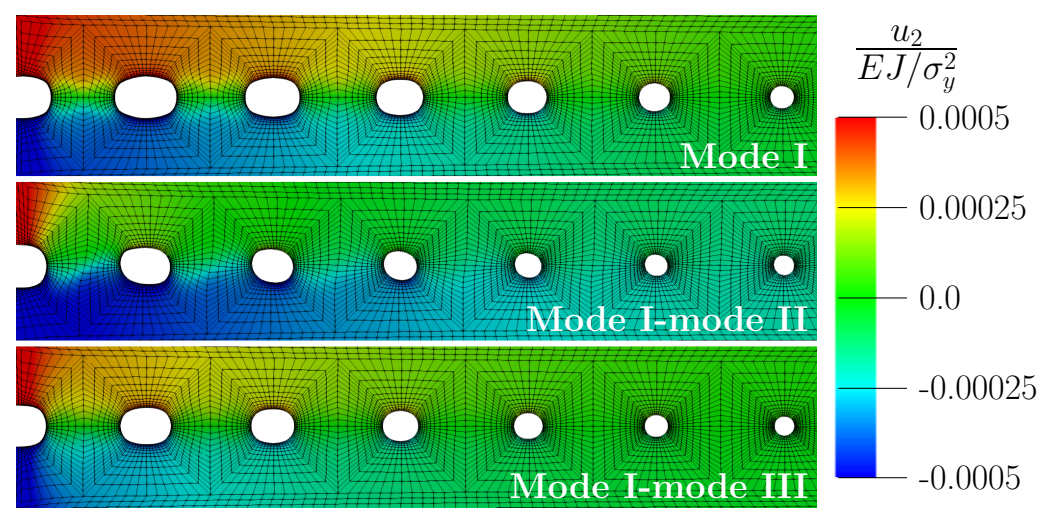

(b)

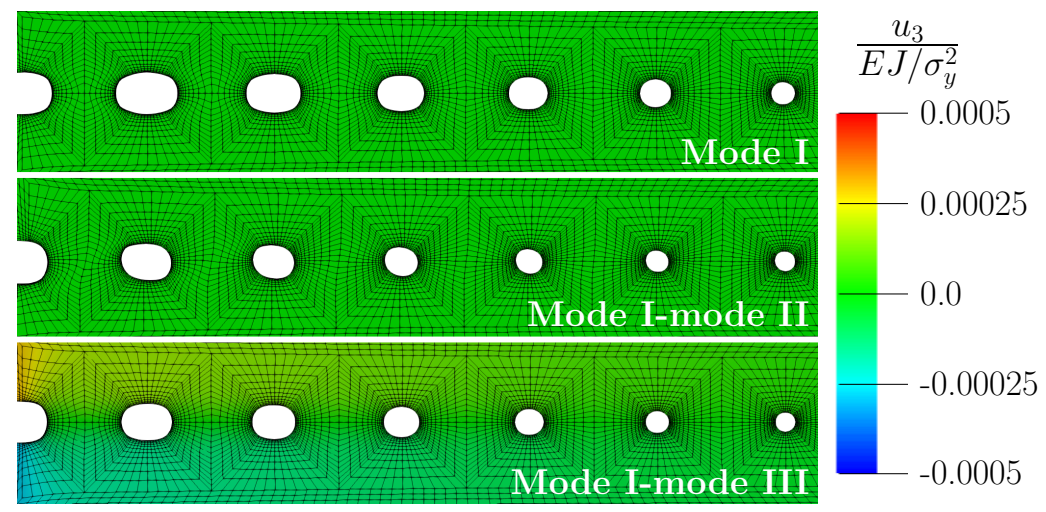

(c)

Figure 6: Contour plots, each comprises the mode I, mode I-mode II $\left(\theta_{\mathrm{II} / \mathrm{I}}=0.2\right)$, and mode I-mode III $\left(\theta_{\text {III } / \mathrm{I}}=0.2\right)$ loading, showing the displacement field components in (a) the $x_{1}$-direction, $u_{1},(\mathrm{~b})$ the $x_{2}$-direction, $u_{2}$, and c) the $x_{3}$-direction, $u_{3}$, at a far-field load intensity of $J /\left(\sigma_{y} X_{0}\right) \approx 0.5$. The model parameters used are $r_{0} / X_{0}=1 / 30$ and $R_{0} / X_{0}=1 / 15\left(f_{0}=0.014\right)$. 


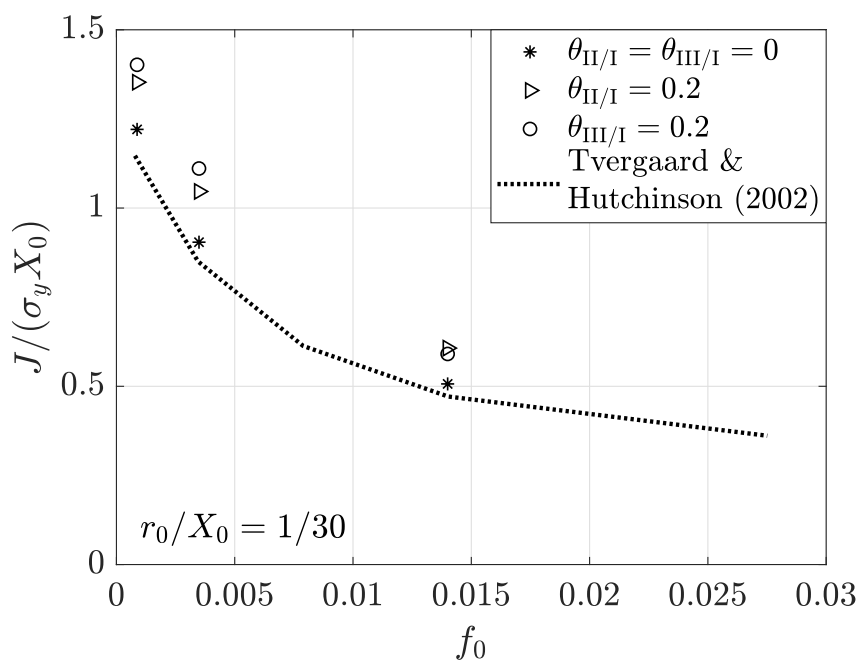

(a)

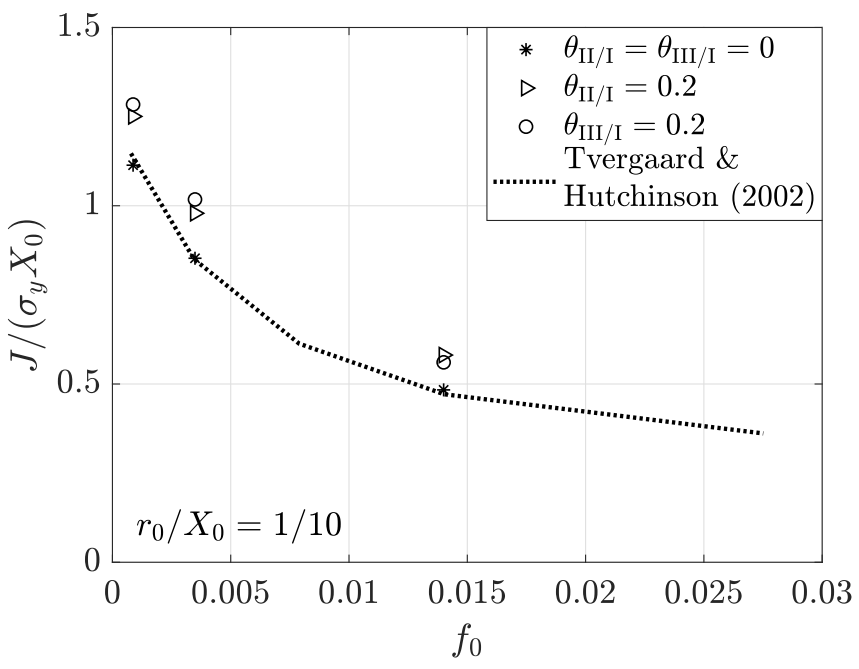

(b)

Figure 7: Applied far-field load intensity, $J /\left(\sigma_{y} X_{0}\right)$, extracted when ligament 1 reaches $\chi / \chi_{0}=0.5$. Here, shown as function of initial void volume fraction and an initial blunt crack tip radius of (a) $r_{0} / X_{0}=1 / 30$ and (b) $r_{0} / X_{0}=1 / 10$. 




Figure 8: Void (1) expansion curves for $r_{0} / X_{0}=1 / 30$ and $R_{0} / X_{0}=1 / 60\left(f_{0}=0.000873\right)$ for different load cases. Results from Tvergaard and Hutchinson (2002) are included for comparison. 


$$
\begin{aligned}
& \rightarrow \theta_{\mathrm{II} / \mathrm{I}}=\theta_{\mathrm{III} / \mathrm{I}}=0 \\
& \triangleright \theta_{\mathrm{II} / \mathrm{I}}=0.1-\triangleright-\theta_{\mathrm{II} / \mathrm{I}}=0.2-\bullet-\theta_{\mathrm{II} / \mathrm{I}}=0.5 \\
& -0-\theta_{\mathrm{III} / \mathrm{I}}=0.1-\ominus-\theta_{\mathrm{III} / \mathrm{I}}=0.2-\bullet \cdot-\theta_{\mathrm{III} / \mathrm{I}}=0.5
\end{aligned}
$$

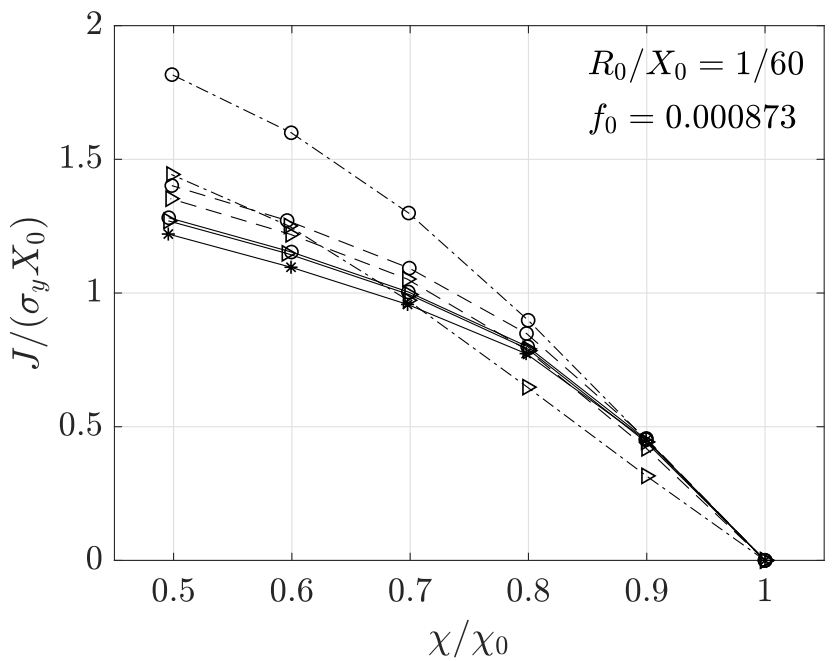

(a)

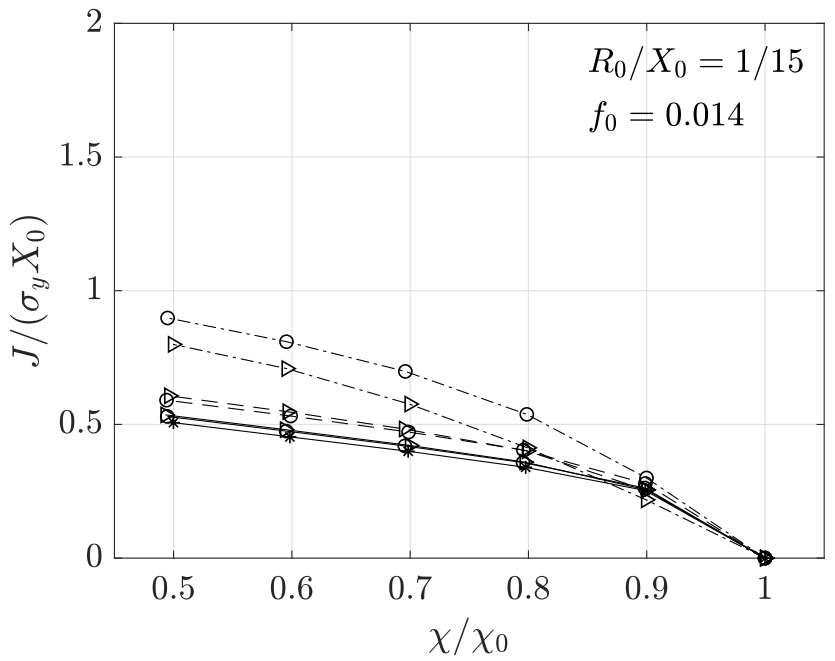

(b)

Figure 9: Applied far-field load intensity, $J /\left(\sigma_{y} X_{0}\right)$, as a function of reduction of ligament 1 for different mode mixity ratios, $\theta$, a fixed initial crack tip radius of $r_{0} / X_{0}=1 / 30$, and two initial void volume fractions, (a) $R_{0} / X_{0}=1 / 60\left(f_{0}=0.000873\right)$ and (b) $R_{0} / X_{0}=1 / 15\left(f_{0}=0.014\right)$. 


$$
\begin{aligned}
& \rightarrow \theta_{\mathrm{II} / \mathrm{I}}=\theta_{\mathrm{III} / \mathrm{I}}=0 \\
& \triangleright \theta_{\mathrm{II} / \mathrm{I}}=0.1-\triangleright-\theta_{\mathrm{II} / \mathrm{I}}=0.2-\diamond-\theta_{\mathrm{II} / \mathrm{I}}=0.5 \\
& -\theta_{\mathrm{III} / \mathrm{I}}=0.1-\ominus-\theta_{\mathrm{III} / \mathrm{I}}=0.2-\bullet \cdot-\theta_{\mathrm{III} / \mathrm{I}}=0.5
\end{aligned}
$$

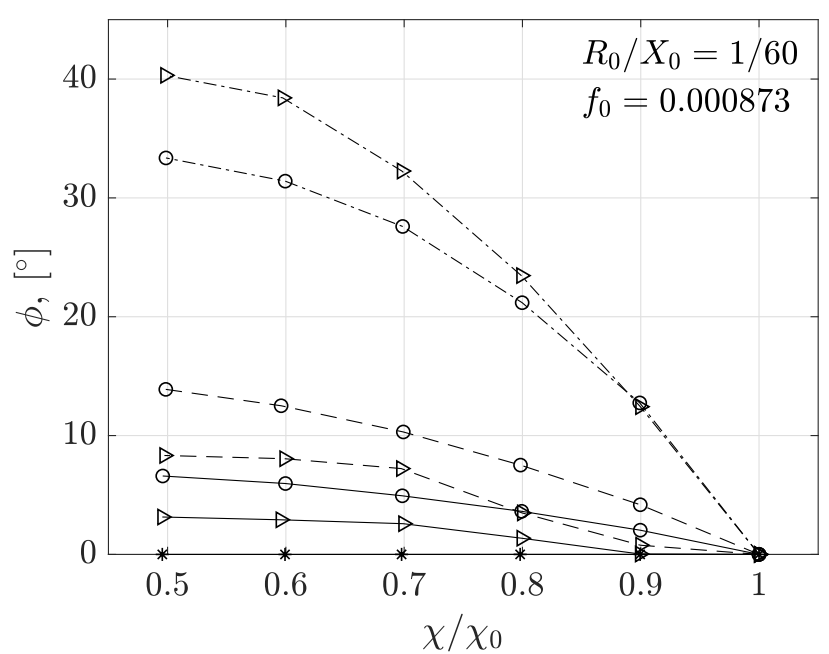

(a)

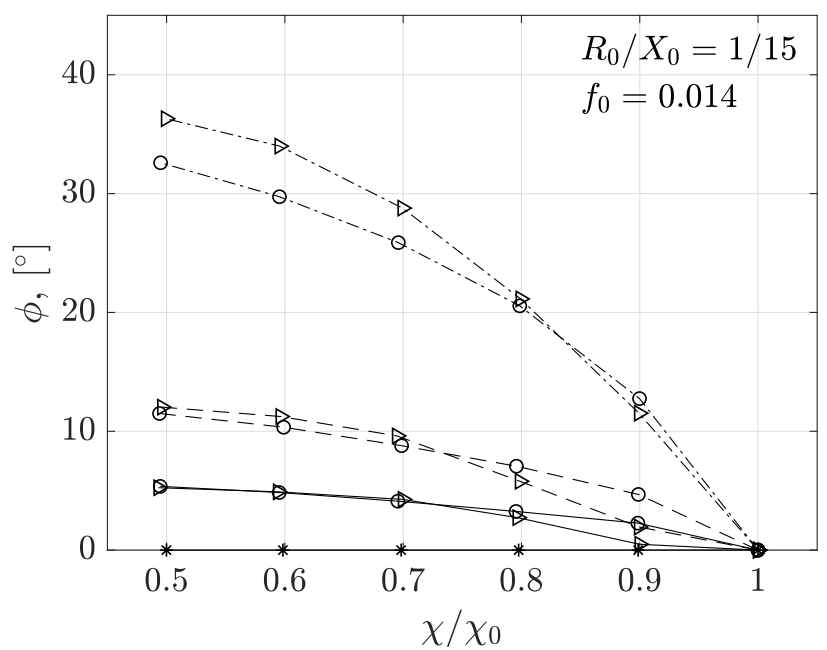

(b)

Figure 10: Void rotation of void (1) as a function of reduction of ligament 1 for different mode mixity ratios, $\theta$ and a fixed initial crack tip radius of $r_{0} / X_{0}=1 / 30$. Here shown for (a) $R_{0} / X_{0}=1 / 60$ $\left(f_{0}=0.000873\right)$ and $(\mathrm{b}) R_{0} / X_{0}=1 / 15\left(f_{0}=0.014\right)$. 

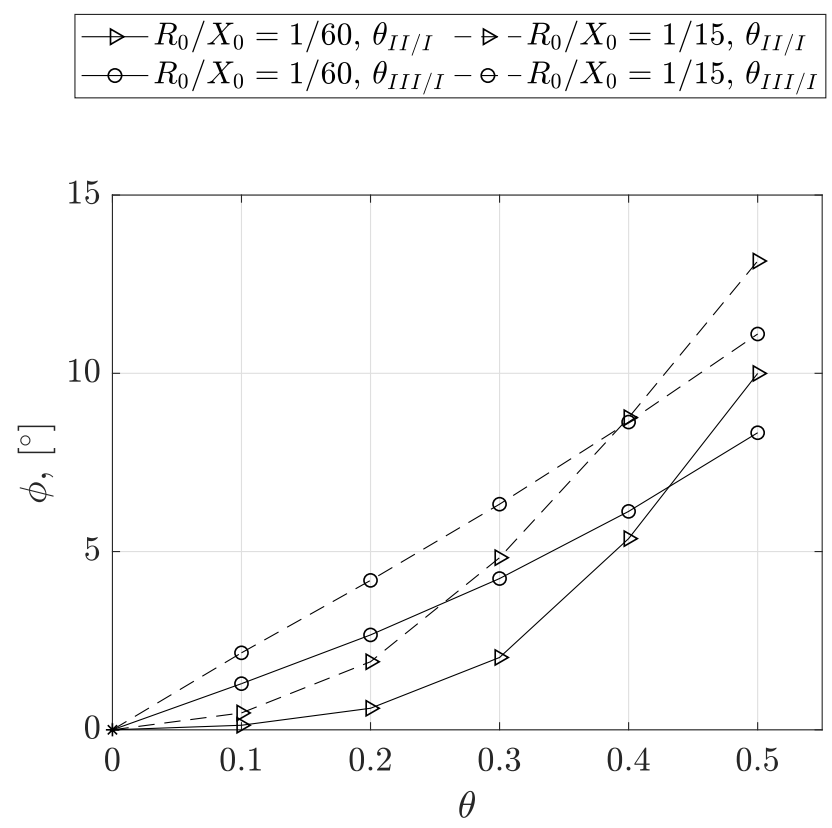

Figure 11: Void rotation of void (1) as a function of mode mixity, $\theta$, for different initial void volume fractions, $R_{0} / X_{0}$, and a fixed initial crack tip radius of $r_{0} / X_{0}=1 / 30$. The values are extracted when the applied far-field load intensity reaches $J /\left(\sigma_{y} X_{0}\right)=0.25$. 


$$
\begin{aligned}
& \rightarrow-\theta_{\mathrm{II} / \mathrm{I}}=\theta_{\mathrm{III} / \mathrm{I}}=0 \\
& \triangleright \theta_{\mathrm{II} / \mathrm{I}}=0.1-\triangleright-\theta_{\mathrm{II} / \mathrm{I}}=0.2-\triangleright-\theta_{\mathrm{II} / \mathrm{I}}=0.5 \\
& \bigcirc-\theta_{\mathrm{III} / \mathrm{I}}=0.1-\ominus-\theta_{\mathrm{III} / \mathrm{I}}=0.2-\ominus \cdot-\theta_{\mathrm{III} / \mathrm{I}}=0.5
\end{aligned}
$$

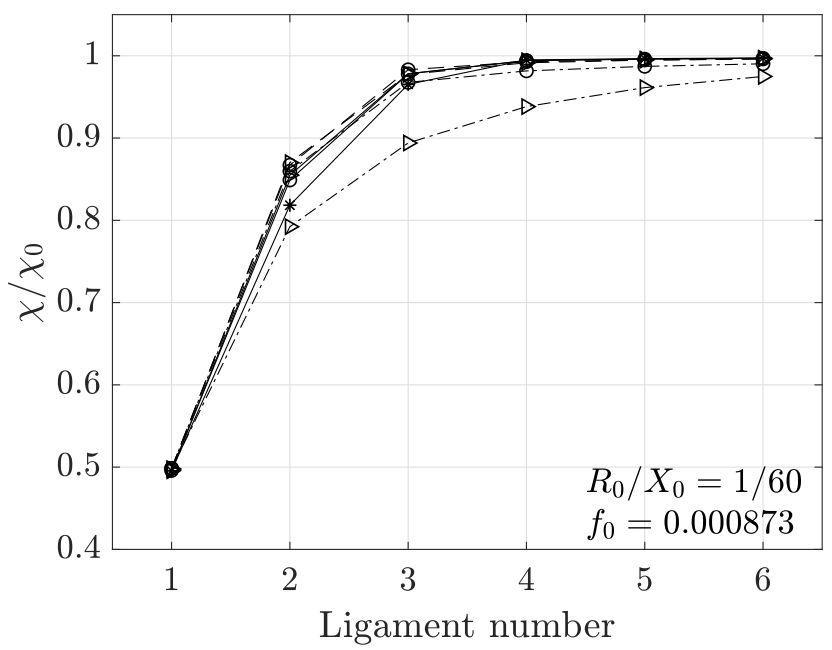

(a)

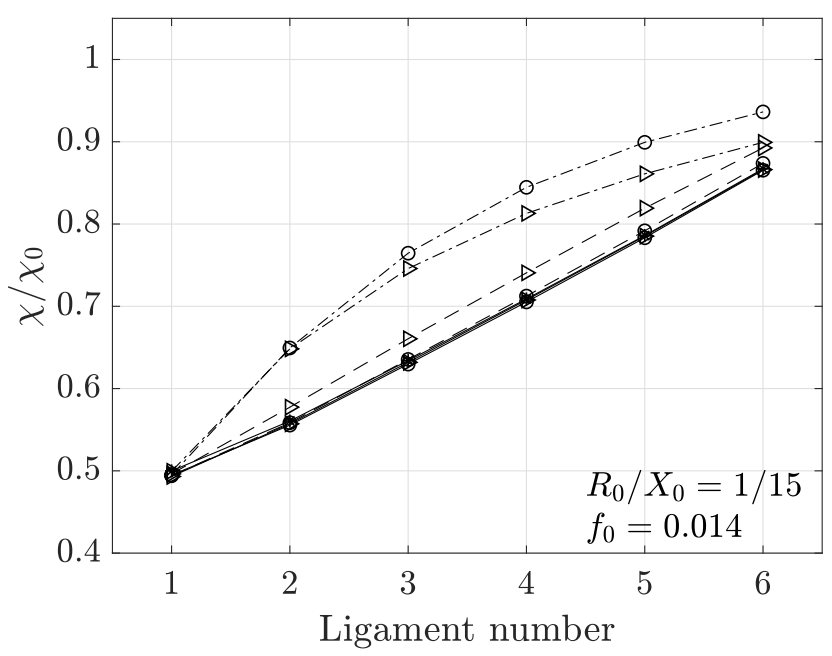

(b)

Figure 12: Ligament reduction of each ligament at the point when ligament 1 has reduced to $\chi / \chi_{0}=$ 0.5 at different mode mixity ratios, $\theta$ and a fixed initial crack tip radius of $r_{0} / X_{0}=1 / 30$. The initial void volume fractions are (a) $R_{0} / X_{0}=1 / 60\left(f_{0}=0.000873\right)$ and (b) $R_{0} / X_{0}=1 / 15\left(f_{0}=0.014\right)$. 


$$
\begin{aligned}
& \rightarrow \theta_{\mathrm{II} / \mathrm{I}}=0.1-\triangleright-\theta_{\mathrm{II} / \mathrm{I}}=0.2-\diamond-\theta_{\mathrm{II} / \mathrm{I}}=0.5 \\
& \bigcirc-\theta_{\mathrm{III} / \mathrm{I}}=0.1-\ominus-\theta_{\mathrm{III} / \mathrm{I}}=0.2-\ominus-\theta_{\mathrm{III} / \mathrm{I}}=0.5
\end{aligned}
$$

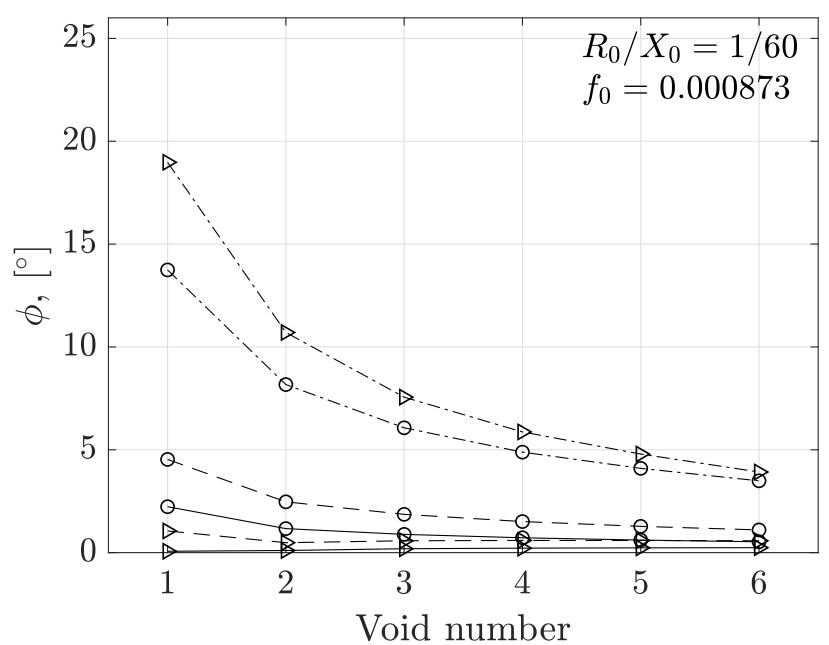

(a)

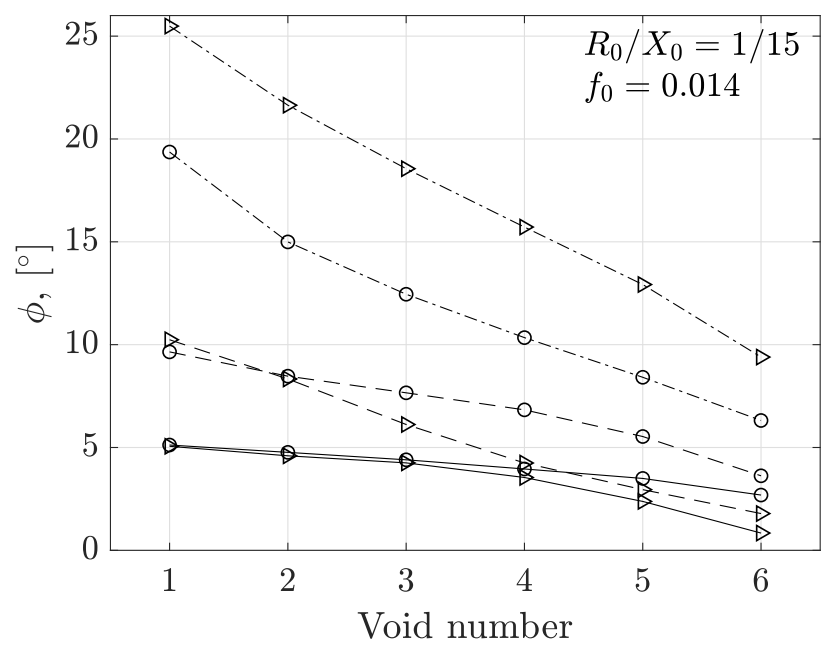

(b)

Figure 13: Void rotation of each row of voids for different mode mixity ratios, $\theta$, at a far-field load intensity of $J /\left(\sigma_{y} X_{0}\right)=0.5$ and a fixed initial crack tip radius of $r_{0} / X_{0}=1 / 30$. Here shown for (a) $R_{0} / X_{0}=1 / 60\left(f_{0}=0.000873\right)$ and (b) $R_{0} / X_{0}=1 / 15\left(f_{0}=0.014\right)$. 